\title{
Media multitasking in adolescence
}

\author{
Matthew S. Cain ${ }^{1}$ - Julia A. Leonard ${ }^{2}$ - John D. E. Gabrieli ${ }^{2,3}$ • Amy S. Finn ${ }^{4}$
}

Published online: 17 May 2016

(C) Psychonomic Society, Inc (outside the USA) 2016

\begin{abstract}
Media use has been on the rise in adolescents overall, and in particular, the amount of media multitasking - multiple media consumed simultaneously, such as having a text message conversation while watching $\mathrm{TV}$ - has been increasing. In adults, heavy media multitasking has been linked with poorer performance on a number of laboratory measures of cognition, but no relationship has yet been established between media-multitasking behavior and real-world outcomes. Examining individual differences across a group of adolescents, we found that more frequent media multitasking in daily life was associated with poorer performance on statewide standardized achievement tests of math and English in the classroom, poorer performance on behavioral measures of executive function (working memory capacity) in the laboratory, and traits of greater impulsivity and lesser growth mindset. Greater media multitasking had a relatively circumscribed set of associations, and was not related to behavioral measures of cognitive processing speed, implicit learning, or manual
\end{abstract}

Electronic supplementary material The online version of this article (doi:10.3758/s13423-016-1036-3) contains supplementary material, which is available to authorized users.

Matthew S. Cain

matthew.s.cain6.civ@mail.mil

1 Cognitive Science Team, U.S. Army Natick Soldier Research, Development, \& Engineering Center, 15 General Greene Avenue, Natick, MA 01760-5000, USA

2 Department of Brain and Cognitive Sciences, Massachusetts Institute of Technology, Cambridge, MA, USA

3 McGovern Institute for Brain Research, Massachusetts Institute of Technology, Cambridge, MA, USA

4 Department of Psychology, University of Toronto, Toronto, Ontario, Canada dexterity, or to traits of grit and conscientiousness. Thus, individual differences in adolescent media multitasking were related to specific differences in executive function and in performance on real-world academic achievement measures: More media multitasking was associated with poorer executive function ability, worse academic achievement, and a reduced growth mindset.

Keywords Media multitasking $\cdot$ Adolescents $\cdot$ Standardized tests $\cdot$ Academic achievement $\cdot$ Working memory $\cdot$ Fluid intelligence

Media multitasking is the act of consuming multiple media simultaneously - for example, having a television on in the background while using a smartphone. Among adolescents, the amount of media multitasking has been increasing, with the average 8- to 18-year-old American spending 29\% of their media consumption time multitasking, up from just $16 \%$ a decade ago (Rideout, Foehr, \& Roberts, 2010). Despite the increase, there is wide variation between individuals, with nearly a fifth of adolescents not reporting any media multitasking (Foehr, 2006). Given both the widespread increase in media multitasking and how much it varies across adolescents, it is important to understand how this behavior relates to fundamental aspects of cognition and measures of academic achievement.

When adults actively consume multiple media, the need to divide attention between information sources can negatively impact processing of the media content, including decreasing reading comprehension (Lin, Lee, \& Robertson, 2011) and increasing receptivity to advertising messages (De Riddere, 2013; Duff, Yoon, Wang, \& Anghelcev, 2014; Kononova, 2013). Beyond the immediate act of media consumption itself, greater self-reported media multitasking has been associated 
with a number of undesirable characteristics in adults, such as a lesser ability to filter out distracting information (Cain \& Mitroff, 2011; Ophir, Nass, \& Wagner, 2009; but see Minear, Brasher, McCurdy, Lewis, \& Younggren, 2013), worse performance on fluid intelligence measures such as Raven's Progressive Matrices (Minear et al. 2013), worse performance on demanding working memory tasks (Ophir et al. 2009), worse performance on task switching (Ophir et al. 2009; but see Alzahabi \& Becker, 2013, and Minear et al. 2013, for conflicting results), and a tendency to use a split rather than unitary focus of attention (Yap \& Lim, 2013). Additionally, heavy media multitasking has been associated with higher levels of impulsivity (Cain \& Mitroff, 2016; Minear et al. 2013), sensation seeking (Duff et al. 2014; Kononova, 2013), attentional lapses, errors, and mind wandering (Ralph, Thomson, Cheyne, \& Smilek, 2014), and even with depression and social anxiety (Becker, Alzahabi, \& Hopwood, 2012).

Only one study to date has directly examined the relationship between cognitive function and media multitasking in adolescents. Greater media multitasking was associated with self-reports of worse executive function in a large sample of adolescents (Baumgartner, Weeda, van der Heijden, \& Huizinga, 2014). Contrary to the self-reports, when assessed experimentally, Baumgartner et al. found evidence for an association between heavier media multitasking and better inhibitory control (i.e., more easily ignoring distractors in an Eriksen flanker task), but no relationships with task switching or working memory performance.

Here we extended understanding about media multitasking in four ways. First, we examined the relationship of media multitasking not only to laboratory measures of cognitive abilities, as in previous studies, but also to real-world measures, specifically academic achievement in reading and math, as measured by statewide testing. Second, we examined a wide range of cognitive and motor abilities and personality traits to discover the scope and limits of the abilities and traits in adolescents that are associated with media multitasking. Third, we included participants from a broad range of ethnic backgrounds and family incomes to ensure a societally relevant picture of the correlates of media multitasking. Finally, we examined these relationships in middle adolescence (around age 14) - an age group that consumes a great deal of media, as compared to their younger and older peers (Rideout et al. 2010), but has received relatively little research attention.

The examination of adolescents allowed us to begin to address the theoretical interpretation of the association of greater media multitasking with worse performance on tests of executive function. On the one hand, greater media multitasking could be the cause of reduced executive abilities. Alternatively, greater multitasking could be a consequence of underlying differences in cognitive abilities. Specifically, those most prone to distraction or impulsive behavior might intentionally media multitask to manage their level of distractibility (Cain \&
Mitroff, 2011). By choosing a distraction they can control (e.g., another form of media), individuals might be less prone to distraction by unpredictable factors (either external stimuli or internal impulses). By examining a younger cohort than had most previous studies, we hoped to shed light on the causal nature of the relationship between media multitasking and cognitive abilities. Specifically, an association between greater media multitasking and lesser executive abilities at younger ages would suggest that greater media multitasking is more likely a consequence than a cause of lesser executive abilities.

We also examined the scope and limits of the correlates of media multitasking in the cognitive and personality domains. In cognition, we examined not only executive functions as indexed by working memory capacity, but also processing speed, implicit learning, and motor (manual) dexterity. In personality, we examined not only impulsivity, but also conscientiousness, grit (or stick-to-it-ness; Duckworth \& Quinn, 2009), and growth mindset (i.e., whether one thinks that intelligence is something that can grow or improve; Dweck, 2006).

We hypothesized that greater media multitasking would be associated with reduced executive function (i.e., reduced working memory capacity), greater impulsivity, and worse scores on statewide achievement tests in reading and math. Research in adults has suggested that greater media multitasking should be negatively associated with these aspects of cognition and impulsivity (Ophir et al., 2009). We predicted that this would also be true for achievement tests, since prior research has linked working memory capacity to academic performance generally (Alloway, Gathercole, Kirkwood, \& Elliott, 2009; Gathercole \& Pickering, 2000), and to scores on statewide achievement tests in particular (Finn et al. 2014). We further hypothesized that media-multitasking behavior would not necessarily be related to cognitive domains that are not as attentionally taxing, such as processing speed, implicit learning, and manual dexterity. With regard to traits and beliefs, we hypothesized that greater grit, conscientiousness, and growth mindset - all of which have been associated with superior academic performance-might be associated with lesser media multitasking.

\section{Method}

\section{Participants}

We administered the Media Use Questionnaire to 74 participants who were recruited from after-school programs and middle schools in the greater Boston area as part of a larger study. Flyers were sent home with 8 th grade students after a brief presentation was given, and interested families contacted the experimenters. All measures analyzed for this study are reported here. One participant was excluded from the analysis for overall poor performance (more than two standard 
deviations below the overall mean on most measures). The remaining 73 participants ranged in age from 12.8 to 16.5 years (mean $=14.4$ years, $S D=0.63$ ) and included 36 females and 37 males. Thirty-three participants identified as AfricanAmerican, 20 as Latino/a, 23 as Caucasian, four as Asian, and two as Native American or Hawaiian (participants could indicate more than one race or ethnicity). Forty-six of the participants qualified for free or reduced-price lunch at some point in the 3 years previous to participation, indicating a family income at or below $185 \%$ of the poverty level $(\$ 42$, 200 per year for a family of four at the time of data collection). Not all participants were able to complete every task due to fatigue or reaching the time limit of their visit (Table 1).

\section{Media-multitasking index}

Participants were given a modified version of the Media Use Questionnaire (Cain \& Mitroff, 2016; Ophir et al. 2009). The questionnaire asked how many hours per week were spent using 12 primary media: watching TV/video/DVDs, watching short video, playing video games, listening to music, listening on nonmusical audio, talking on the phone (or voice chat), talking face to face (or video chat), instant or text messaging, reading print media, reading electronic media, writing (either electronic or longhand), and creating nonwritten arts and crafts. For each primary medium, participants were asked how often they also engaged with each of the media as a secondary task, with possible responses of "Never" (coded as 0$)$, "A little of the time" (1/3), "Some of the time" (2/3),

Table 1 Participant counts, means, and standard deviations for each measure

\begin{tabular}{llrr}
\hline Measure & \#Participants & Mean & \multicolumn{1}{c}{$S D$} \\
\hline Media-Multitasking Index & 73 & 3.00 & 1.90 \\
MCAS Math & 73 & 246.22 & 17.16 \\
MCAS English & 73 & 248.77 & 11.06 \\
Count span & 69 & 2.86 & 1.88 \\
$N$-back & 58 & 0.66 & 0.13 \\
Filtering & 58 & 0.01 & 0.11 \\
Comprehension & 69 & 11.74 & 3.05 \\
Vocabulary & 69 & 11.29 & 2.85 \\
Calculation & 67 & 105.27 & 15.79 \\
Growth mindset & 70 & 4.00 & 1.33 \\
Grit & 70 & 3.20 & 0.66 \\
Conscientiousness & 69 & 3.35 & 0.55 \\
DSIS-C (impulsivity) & 70 & 2.66 & 0.98 \\
Processing speed & 64 & 19.95 & 6.00 \\
Pegboard (dexterity) & 67 & 52.63 & 8.74 \\
Probabilistic classification task & 70 & 0.60 & 0.12 \\
\hline
\end{tabular}

For the measures other than filtering and pegboard, a higher score indicates better performance or more of the measured trait and "Most of the time" (1). For each primary medium, the 12 secondary media ratings were summed and the total multiplied by the number of hours per week that the primary medium was used. These 12 weighted values were summed and divided by the total number of primary media usage hours. This resulting Media-Multitasking Index (MMI) ranged from 0 (all media consumed singly) to 12 (all media consumed simultaneously with other media). The normalization for total media consumption meant that, for example, an individual who plays $40 \mathrm{~h}$ of video games per week could still have a low MMI if they consume no other media simultaneously with the games.

\section{Academic performance}

We obtained Massachusetts Comprehensive Assessment System (MCAS) Math and English Language Arts scores from databases maintained by the Massachusetts Department of Elementary and Secondary Education. In all cases, the scaled scores that participants received in the year 2012 (regardless of whether they were in 7th or 8th grade) were used as the primary measures. Scores ranged from 200 to 280 . The Math subtest included algebra, geometry, probability, and measurement questions and allowed the use of a calculator for some portions. The English Language Arts subtest involved reading multiparagraph passages and answering questions related to the meaning and vocabulary of the passages. Both subtests were presented in written form in two sessions each and were administered in a classroom setting. To view example questions from this test, visit http://www.doe.mass. edu/mcas/testitems.html?yr=12.

\section{Executive function measures}

Three measures of executive function were assessed: working memory count span, working memory $n$-back, and working memory filtering.

Working memory count span The count span task (Case, Kurland, \& Goldberg, 1982; Cowan et al. 2005) was administered via computer using the PsychoPy software (Peirce, 2007). Participants viewed an array with blue circles, blue triangles, and red circles, and were instructed to count only the blue circles (targets). When they were done counting, they were instructed to press the space bar to move on (the program would time out after $5 \mathrm{~s}$ ). After one or more arrays were presented, participants entered the number of targets presented in each display in the order that they had been presented. Participants saw a total of three instances of each working memory load (one to six consecutive arrays), which were presented in random order. 
Working memory N-back In this task (Kane, Conway, Miura, \& Colflesh, 2007; Owen, McMillan, Laird, \& Bullmore, 2005), participants were presented with letters one at a time and asked to indicate with a button press if the current letter was the same as the letter that had been presented $n$ screens previously. Prior to each block of trials, participants were instructed whether to look for a match at $n=1,2$, or 3 back. Participants also performed a baseline target detection ("0-back") task in which they were asked to press a key whenever they saw the letter "w." For all task blocks, the letters were drawn from a pool of eight (b, f, h, j, q, m, r, and w) and were presented in both upper- and lowercase to prevent matching on the basis of purely perceptual features. Each letter was presented in the middle of an array of empty circles for $500 \mathrm{~ms}$ and was followed by $2,500 \mathrm{~ms}$ of fixation before the next letter was presented. Participants had until the end of the fixation $(2$, $900 \mathrm{~ms}$ total) to respond on each trial. Thirty percent of all trials were targets (i.e., repetitions requiring an affirmative response). Each block contained ten letters and began with an initial fixation of $500 \mathrm{~ms}$ and an instruction screen that indicated the block type for 3,000 ms. Each block was followed by $12 \mathrm{~s}$ of rest, during which the screen showed a fixation cross on a black background. Participants completed two runs of the $n$-back task, while in an MRI scanner, with each run containing 12 blocks. Sensitivity (hits minus false alarms) was computed separately for each load (0-3) and then averaged into a composite score.

Working memory filtering Participants were first presented with a cue shape (triangle or square) for 3-5 s, which indicated whether or not they should filter subsequent information entering working memory. If the cue was a triangle (shape counterbalanced across participants), they were asked to attend only to the red items in the subsequent display and to ignore yellow items. If the cue was a square, they were instructed to attend to all items on the subsequent display (i.e., both yellow and red). Next, participants viewed an array of 16 circles arranged in one large circle for $1 \mathrm{~s}$. Each of the small circles could be filled with either a red or a yellow star; in any given array, either two or four stars were present. After a variable delay (2-4 s), participants were asked to decide whether one of the stars that they had been asked to attend to was present in the circle indicated with a question mark probe. This design contained three trial types: distraction trials (four stars were present, but two should be ignored), high mnemonic load trials (four stars were present, all to be held in mind), and low mnemonic load trials (two stars were present, all to be held in mind). Across all trial types, half of the probes appeared in a target position and half occurred in a nontarget location adjacent to one of the target positions. On distraction trials, nontarget probes occurred in one of the distractor positions half of the time. Participants completed a total of 120 trials, presented in four blocks of 30 trials each, while in an MRI scanner. Across the experiment, 40 trials apiece were presented of each of the distraction, high-load, and low-load trial types. The primary measure of interest was the difference between performance on the low-load and distraction trials (i.e., the two conditions with a memory load of two items).

\section{Crystallized intelligence}

Verbal comprehension The Comprehension and Vocabulary subtests of the Wechsler Intelligence Scale for Children, Fourth Edition (WISC; Wechsler, 2003) were administered. For the Comprehension subtest, participants verbally explained familiar situations and actions (e.g., "Why do we turn out lights when we leave a room?"). For the Vocabulary subtest, participants were shown pictures and asked to name the objects and presented words aloud and asked to define them. Both subset scores were converted into scaled scores in accordance with the testing manual.

Calculation In the Calculation subtest of the WoodcockJohnson III Tests of Achievement, participants were given 45 calculation problems in mathematics. The problems began with easy arithmetic and got progressively more difficult, up to calculus integration problems. Test administration was ended after six incorrect answers were given in a row, and the raw number of correct responses was converted into a scale score in accordance with the testing manual.

\section{Personality factors}

Growth mindset This scale is designed to measure participants' implicit theories of intelligence - that is, whether they believed that their intelligence or ability was malleable and could improve with effort, rather than being a set factor beyond their control (Blackwell, Trzesniewski, \& Dweck, 2007; Dweck, 2006). The three items (e.g., "You have a certain amount of intelligence, and you really can't do much to change it.") were rated on a scale of 1 (strongly agree) to 6 (strongly disagree). A mean Growth Mindset score was calculated, with low scores representing the view that intelligence cannot be changed and high scores representing a growth mindset, or the view that they can.

Grit The Grit short scale was used to measure participants' ability to persevere in difficult or even impossible tasks. This scale consists of eight questions about perseverance and passion for long-term goals (Duckworth \& Quinn, 2009). Questions were answered on a 5-point Likert scale, from 1 (very much like me) to 5 (not like me at all). The mean of all items was used as the Grit score, with a lower score indicating a lower self-perception of Grit. 
Conscientiousness The Big Five Personality Inventory is a widely used personality questionnaire that measures openness, conscientiousness, extraversion, agreeableness, and neuroticism (John \& Srivastava, 1999; Soto \& John, 2012). This scale includes 44 statements (e.g., "I see myself as someone who does a thorough job"), which are ranked on a 5-point Likert scale from 1 (strongly disagree) to 5 (strongly agree). In particular, we were interested in the Conscientiousness factor because it, like Grit, is thought to reflect task perseverance.

\section{Domain-specific impulsivity in school-age children (DSIS-C)} Participants were asked to complete this eight-item questionnaire designed to measure their impulsivity in a school-type setting using common and consequential situations (Tsukayama, Duckworth, \& Kim, 2013). For example, a student would be asked to indicate the frequency of "I forgot something I needed for class" as: almost never, about once a month, about two to three times a month, about once a week, or at least once a day. Higher scores indicate more impulsive behavior.

\section{Other tasks}

Cognitive processing speed We administered the Coding and Symbol Search subtests from the WISC. On the Coding subtest, participants were asked to translate digits into symbols by referring to a corresponding digit-symbol key (nine novel symbols corresponded to the digits 1 through 9). On the Symbol Search subtest, participants were asked to indicate whether either of two symbols on the left side of a page matched any of five symbols on the right side of a page. Participants had 2 min to complete each task, and the number of items completed correctly during this time was the dependent measure for each task. These measures were merged to yield a standardized processing speed score, in accordance with WISC scoring procedures.

Manual dexterity (Peg board) The Lafayette pegboard (Lafayette Instrument Co., Lafayette, IN) was administered to measure manual dexterity and coordination. Participants were told to only use their dominant hand to place 25 pegs into 25 holes on a $5 \times 5$ grid in a left-to-right manner (row by row) as quickly and efficiently as possible. The completion time in seconds served as the dependent measure.

Probabilistic classification In the weather prediction task, participants were asked to guess whether an array of cards was more likely to be associated with sun or rain (Gluck, Shohamy, \& Myers, 2002; Knowlton, Squire, \& Gluck, 1994; Shohamy, Myers, Hopkins, Sage, \& Gluck, 2008). At first, participants were made to guess, but as the experiment went on they were able to learn from probabilistic feedback, in the form of smiling or frowning faces appearing after a response was made. The stimuli consisted of four unique cards, displayed in 14 combinations. These combinations were composed of either a single card, two cards at the same time, or three cards at the same time; all four cards were never simultaneously presented. Each card combination was probabilistically associated with an outcome of rain or sun (ranging from $10 \%$ to $90 \%$ ). Responses over the entire 100 trials were scored for optimal accuracy - that is, whether the participant responded with the more likely outcome for each display.

\section{Results}

\section{Media}

Media usage Overall, participants reported consuming a great deal of media, with a mean total primary-medium consumption of $149 \mathrm{~h}$ per week (median $=128 \mathrm{~h} /$ week), but this value ranged from 9 to $583 \mathrm{~h} /$ week. Many participants estimated consuming media well in excess of $168 \mathrm{~h} /$ week, suggesting a systematic overestimation of media usage, limiting the interpretability of the raw usage values for each medium, but not necessarily of the MMI, which was normalized by total media use. The most heavily used primary medium was television, with a mean report of $12 \mathrm{~h} /$ week (median $=7 \mathrm{~h} /$ week, range $=0-90$ ).

Media multitasking index The MMI was distributed unimodally (Fig. 1), with a rightward skew (skewness $=0.864$ ). The mean MMI of 3.00 represents about $25 \%$ concurrent media usage $($ median $=2.69$, range $=0.05-9.11)$. Within the narrow age range of this sample, we observed no relationship between age and MMI $(r(71)=-.05, p=.652,95 \% \mathrm{CI}=$ $[-.28, .18])$. Females had a greater mean MMI $(3.24, S D=$ 1.77) than did males $(2.77, S D=2.01)$, but this difference was not significant $[t(71)=1.06, p=.29, d=0.25]$. Those who had received free or reduced-price lunch had higher MMI scores than did those not receiving lunch subsidies

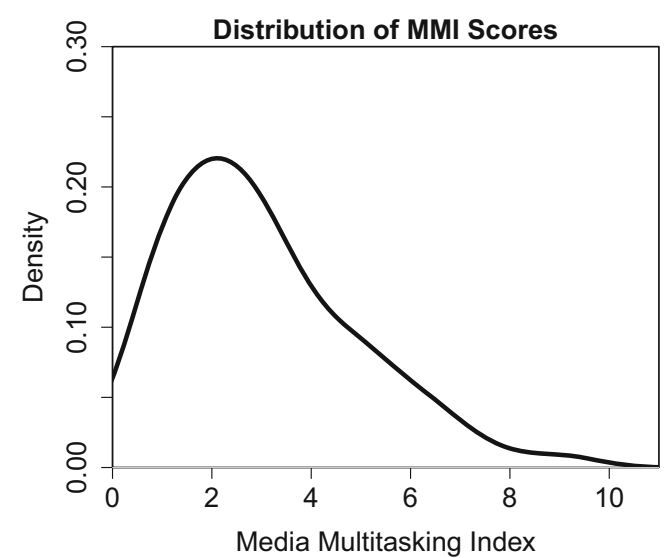

Fig. 1 Kernel density plot of Media-Multitasking Indices for all participants (possible range: $0-12$ ) 
(3.35 vs. 2.40$)[t(70.88)=2.39, p=.020, d=0.54$; degrees of freedom were Welch-corrected for unequal variances].

\section{Massachusetts comprehensive assessment system}

More media multitasking was associated with worse performance on academic assessments. MMI scores were significantly correlated with MCAS scores for both the Math $(r(71)=-.30, p=.011, \mathrm{CI}=[-.49,-.07])$ and English Language Arts $(r(71)=-.29, p=.012, \mathrm{CI}=[-.49,-.07])$ subtests (Fig. 2).

\section{Executive functions}

Count span MMI scores correlated significantly with the count $\operatorname{span}(r(67)=-.27, p=.024, \mathrm{CI}=[-.48,-.04])$ measure of working memory capacity, with more media multitasking being associated with lower capacity.

N-back MMI scores correlated significantly with overall $n$ back performance (hits minus false alarms, averaged across all levels of $n)(r(56)=-.38, p=.003, \mathrm{CI}=[-.60,-.13])$, with more media multitasking being associated with worse performance (see Fig. 3). Further details about each working memory load are available in the supplementary information.

Filtering Filtering performance was measured as the difference in accuracy between low-mnemonic-load trials (two targets, no distractors) and distraction trials (two targets, two distractors). No significant correlation was apparent between MMI scores and working memory filtering performance $(r(56)=-.05, p=.704, \mathrm{CI}=[-.31, .21])$.

\section{Crystallized intelligence}

Verbal comprehension We did not find significant correlations between MMI scores and performance on WISC Comprehension $(r(67)=-.21, p=.078, \mathrm{CI}=[-.43, .02])$ or

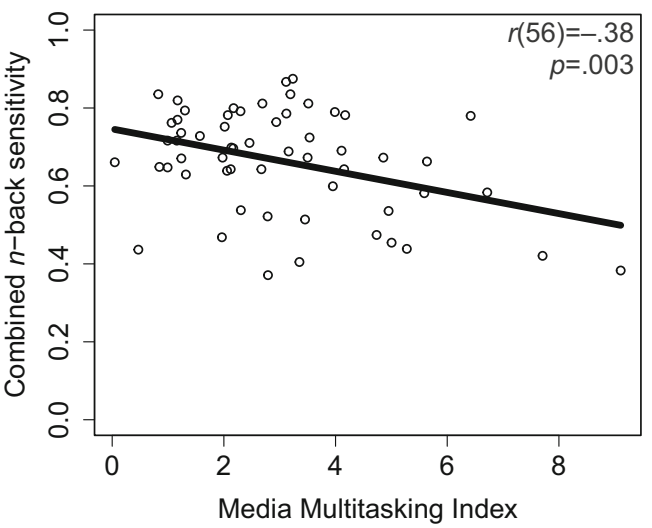

Fig. 3 Correlations between the Media-Multitasking Index and $N$-back hits minus false alarms (averaged across conditions)

WISC Vocabulary $(r(67)=-.17, p=.166, \mathrm{CI}=[-.39, .07])$. There were significant correlations, however, between MCAS English Language Arts performance and both WISC Comprehension $(r(67)=.62, p<.001, \mathrm{CI}=[.45, .75])$ and WISC Vocabulary $(r(67)=.74, p<.001, \mathrm{CI}=[.62, .84])$. See the supplementary information for a table of the correlations between all measures.

Calculation No significant correlation emerged between MMI scores and Woodcock-Johnson calculation performance $(r(65)=-.09, p=.46, \mathrm{CI}=[-.33, .15])$, but the correlation between MCAS Math performance and calculation performance was significant $(r(65)=.78, p<.001, \mathrm{CI}=[.66, .86])$.

\section{Personality factors}

Growth mindset MMI scores significantly correlated with growth mindset $(r(68)=-.34, p=.004, \mathrm{CI}=[-.53,-.12]$; Fig. 4), with more media multitasking being associated with less of a belief that intelligence is malleable.

Grit We found no significant correlation between MMI scores and Grit scores $(r(68)=-.10, p=.424, \mathrm{CI}=[-.32, .14])$.
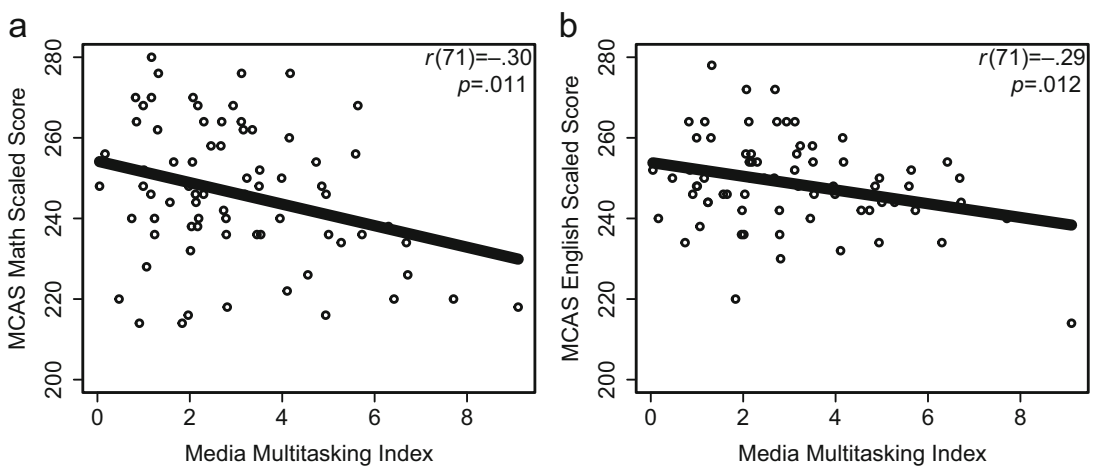

Fig. 2 Massachusetts Comprehensive Assessment System scaled scores for (A) Math and (B) English Language Arts, both negatively correlated with the Media-Multitasking Index. Lines represent best-fit linear regressions 


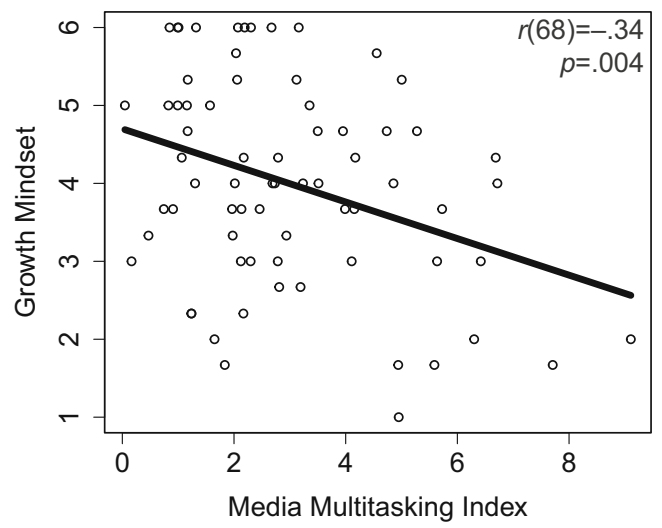

Fig. 4 Growth mindset is negatively correlated with the MediaMultitasking Index (MMI), with lower MMI scores being associated with believing that intelligence is more malleable and higher MMI scores associated with believing intelligence is less malleable

Conscientiousness There was no significant correlation of MMI scores with the Conscientiousness factor of the Big Five Personality Inventory $(r(67)=-.03, p=.803, \mathrm{CI}=[-.27$, $.21]$ ), or with any of the other four factors (all $|r| \mathrm{s}<.15$, all $p \mathrm{~s}>.3$ ).

\section{Domain-specific impulsivity in school-age children MMI} scores correlated significantly with DSIS-C behavior scores $(r(68)=.25, p=.039, \mathrm{CI}=[.01, .46])$, with more media multitasking being associated with more impulsive behaviors reported.

\section{Other tasks}

There was no relationship between MMI scores and the scaled measures of cognitive processing speed $(r(62)=.03, p=.796$, $\mathrm{CI}=[-.21, .28]$ ), manual dexterity (i.e., performance on the peg board task; $r(65)=-.11, p=.361, \mathrm{CI}=[-.34, .13])$, or performance on the probabilistic classification task $(r(68)=$ $-.07, p=.580, \mathrm{CI}=[-.30, .17])$.

\section{Discussion}

Media multitasking has been on the rise in adolescents, but its relationships to cognition, scholastic achievement, and personality have not been assessed. Here, we found that increased media multitasking was linked to poorer performance on measures of academic performance, poorer performance on laboratory measures of working memory capacity (executive function), more self-reported impulsive behavior, and lesser growth mindset. These relations were specific, in that they did not extend to other kinds of cognitive or perceptual-motor performance or other personality measures. These results demonstrate that the connections between media multitasking and specific individual differences in executive functions and impulsiveness form well before adulthood.

One novel finding was that greater media multitasking correlated with worse statewide standardized test scores measuring academic achievement in math and English. These tests have real-world impacts for schools as measures of student proficiency, and a great deal of effort is put toward improving performance. Our results show that participants' media consumption patterns outside of school are related to their performance on these consequential tests in the classroom. However, we caution that because of the difficulty in establishing the direction of causality (e.g., that media multitasking may be a consequence of underlying cognitive differences), simple interventions such as regulating the amount that adolescents multitask their media consumption may not increase test scores. The absence of significant correlations between media multitasking and laboratory measures of vocabulary and calculation abilities adds further caution against expecting large changes in performance. Still, future research with larger samples that systematically explore behavioral interventions could shed light on any such causal link.

\section{Executive functions}

One explanation for the media-multitasking related differences seen in standardized test scores across the participants in our study may be underlying differences in executive functions. In line with findings in adults (e.g., Minear et al., 2013; Ophir et al. 2009), we found a strong negative relationship in adolescents between the amount of self-reported media multitasking and performance on laboratory executive function measures. Notably, not all aspects of cognition showed the same pattern. Executive functions that involved processing information while holding items in working memory (count span and $n$-back) were related to media multitasking, whereas other measures, such as cognitive processing speed, were not.

Media multitasking involves considerable switching of attention between multiple sources of information (Brasel \& Gips, 2011), a situation that should put large demands on working memory updating. According to this view, greater media multitasking could train and enhance working memory abilities, just as extensive action videogame experience appears to enhance particular executive control and attentional abilities (e.g., Cain, Prinzmetal, Shimamura, \& Landau, 2014; Hubert-Wallander, Green, \& Bavelier, 2011; Strobach, Frensch, $\&$ Schubert, 2012). However, the present data suggest that precisely those executive functions that would be most useful for repeatedly switching focus are inversely correlated with habitual simultaneous media consumption.

The finding that adolescents reporting greater media multitasking have lesser executive abilities aligns with a previous finding in adults that both greater media multitasking and selfperceived multitasking ability correlated negatively with a 
behavioral measure of multitasking that placed strong demands on working memory (Sanbonmatsu, Strayer, Medeiros-Ward, \& Watson, 2013). Although it is not possible to determine the causal nature of the relationship between media multitasking and executive function abilities from correlational analyses, the consistent finding that those who habitually multitask media are also those least able to effectively multitask, starting at least by middle adolescence, supports the idea that differences in underlying cognitive abilities may drive simultaneous media consumption behaviors. Furthermore, we observed a negative relationship between the MMI and impulsivity-also noted in adults (Cain \& Mitroff, 2016) - which suggests that media multitasking might be a manifestation of underlying differences in inhibitory control.

One possible way that differences in inhibitory control could manifest in media multitasking is as an attentional "insurance policy" (Cain \& Mitroff, 2016), with those individuals most susceptible to distraction learning (implicitly or explicitly) to create manageable distractions. For example, listening to familiar music on headphones while reading in a café would demand some attention, but might be more predictable and easier to disengage from than the conversations at nearby tables. Thus, consuming another form of media could perhaps lead to more overall engagement with the primary medium than would occur otherwise.

The relationship seen here between media multitasking and working memory stands in contrast to the results of Baumgartner et al. (2014), who found no relationship between MMI and working memory. This difference may reflect the extent to which different working memory measures indexed the executive aspects of working memory. Baumgartner et al. measured working memory by a test of digit span, which is thought to involve little if any executive function. We measured working memory by tests of complex working memory capacity that place great demands on executive functions by requiring the simultaneous holding of information in mind while performing another task (counting and encoding subsequent stimuli; Engle, Tuholski, Laughlin, \& Conway, 1999). Thus, greater media multitasking may be specifically related to a reduction in the executive function component of working memory.

Overall, the present $n$-back task findings are consistent with those of Ophir et al. (2009). However, Ophir et al. reported MMI-related differences in false alarm rates, whereas in the present experiment, MMI predicted differences in hit rates but not false alarm rates (see the supplementary information), leading to somewhat different interpretations. Ophir et al. argued for an inhibitory deficit leading to false alarms through impulsive responses to familiar items, but our results are more consistent with the interpretation that participants are missing targets because they have not properly maintained and/or updated their working memory. The overall methods of the two $n$-back tasks were similar, with the primary differences being the younger age of the present participants and the testing taking place inside an MRI scanner (potentially more distracting than a normal laboratory). Also we used blocks of ten letters, whereas Ophir et al. used blocks of 30 letters, which may have increased the potential for intrusion errors. Given these results, further investigation with both the $n$-back and other tasks will be needed to understand the true nature of the relationship between working memory and media multitasking.

The present working memory filtering result initially appears to be at odds with the MMI-related differences reported by Ophir et al. (2009), who found that filtering in a working memory context was negatively associated with MMI. However, the filtering tasks employed across the two studies were very different. The task in the present study was designed for younger children and had only one relatively low filtering load (two targets and two distractors). In the Ophir et al. report, they found no differences between adult heavy and light media multitaskers with this same load (only two distractors to be filtered). Indeed, they only observed filtering-related differences when participants were asked to filter loads of four and six. Thus, a larger filtering load is likely necessary to expose media-multitaskingrelated differences in adolescents.

One factor that warrants further scrutiny is socioeconomic status (SES). Lower SES has previously been associated with poorer academic performance and executive function (Farah et al. 2006; Hackman, Gallop, Evans, \& Farah, 2015; Lawson $\&$ Farah, 2015; Mackey et al., 2015) and increased media use (Rideout et al., 2010), but not with media multitasking (Foehr, 2006). In the present sample, our indicator of SES was whether or not a participant had received free or reduced-price school lunch, with nearly two-thirds having done so. We reran our key analyses within this lower-SES group and found the same overall pattern of correlations, although not all relationships remained significant, given the reduced power. Future studies with more participants and continuous rather than binary SES measurements may be able to tease apart the interplay between these factors.

\section{Crystalized intelligence}

The lack of a significant correlation between media multitasking and the crystallized intelligence measures was somewhat surprising, given multitasking's significant correlation with academic measures. Although there were strong correlations between performance on the crystallized intelligence measures and their counterpart academic measures, the two types of tests differed in a few key ways. For example, the two WISC verbal comprehension tests were composed of short items presented orally, whereas MCAS involved reading long passages. Similarly, the calculation test was made up of straightforwardly presented pencil-and-paper calculation problems, whereas the MCAS test focused on word problems, 
graph reading, and understanding geometric figures; adolescents were also permitted to use calculators when taking the MCAS math. Additionally, all crystallized intelligence measures were administered singly in the lab, whereas the MCAS was administered as a group in a standard classroom setting. Thus, it may be that media multitasking is not strongly related to verbal or mathematical ability per se, but more to other skills helpful in academic situations - for example, the ability to manipulate complex question presentations in order to extract the information needed to make content judgments and perform calculations, or the ability to maintain focus on a demanding task in a potentially distracting classroom setting.

\section{Personality and factors}

Given the associations between scholastic achievement and growth mindset, grit, and conscientiousness, we initially hypothesized that these traits would also be associated with media multitasking. Indeed, greater growth mindset, like better academic performance, was associated with lesser media multitasking. There were, however, no relationships between media multitasking and either grit or conscientiousness. Although our limited sample size does not allow us to rule out the existence of weak correlations, we did observe the expected positive correlations between grit and conscientiousness and the expected negative correlations between impulsivity and both grit and conscientiousness (see the supplementary information).

This pattern of findings may be related to differences in how these personality and belief dimensions relate to academic outcomes. Growth mindset has been associated with academic achievement in terms of both grades (Blackwell et al., 2007) and standardized test scores (Good, Aronson, \& Inzlicht, 2003). In contrast, grit and conscientiousness may reflect motivational factors that are captured by grades, but not by test scores (Duckworth, Quinn, \& Tsukayama, 2012; but see West et al. 2016).

\section{Other tasks}

We included three measures in the analysis that were not predicted to correlate with media multitasking: cognitive processing speed, manual dexterity, and probabilistic classification. Indeed, none was significantly associated with media multitasking, suggesting that variation in media multitasking is more strongly related to executive functions than to other forms of ability, such as processing speed, physical dexterity, or procedural learning.

\section{Conclusions}

In sum, we found a link between greater media multitasking and worse academic outcomes in adolescents. This relationship may be due to decreased executive functions and increased impulsiveness - both previously associated with both greater media multitasking and worse academic outcomes. Greater media multitasking was associated with specific cognitive and personality factors, and not with multiple other factors. These results extend previous findings from adults and suggest that the relationships between cognitive abilities and media multitasking are established by middle adolescence.

Author note This work was funded by the Bill and Melinda Gates Foundation (through a grant to J.D.E.G. and C.F. O. Gabrieli) and the National Institutes of Health (NRSA grant to A.S.F.). We thank John Salvatore, Jennifer Minas, Calvin Goetz, Brian Chan, and Micah Nishigaki for help with data collection, and Martin West, Jon Fullerton, and Jared Silver for help obtaining test scores.

\section{References}

Alloway, T. P., Gathercole, S. E., Kirkwood, H., \& Elliott, J. (2009). The cognitive and behavioral characteristics of children with low working memory. Child Development, 80, 606-621. doi:10.1111/j.14678624.2009.01282.x

Alzahabi, R., \& Becker, M. W. (2013). The association between media multitasking, task-switching, and dual-task performance. Journal of Experimental Psychology: Human Perception and Performance, 39, 1485-1495. doi:10.1037/a0031208

Baumgartner, S. E., Weeda, W. D., van der Heijden, L. L., \& Huizinga, M. (2014). The relationship between media multitasking and executive function in early adolescents. Journal of Early Adolescence, 34, 1120-1144. doi:10.1177/0272431614523133

Becker, M. W., Alzahabi, R., \& Hopwood, C. J. (2012). Media multitasking is associated with symptoms of depression and social anxiety. Cyberpsychology, Behavior, and Social Networking, 16, 132-135. doi:10.1089/cyber.2012.0291

Blackwell, L. S., Trzesniewski, K. H., \& Dweck, C. S. (2007). Implicit theories of intelligence predict achievement across an adolescent transition: A longitudinal study and an intervention. Child Development, 78, 246-263. doi:10.1111/j.1467-8624.2007.00995.x

Brasel, S. A., \& Gips, J. (2011). Media multitasking behavior: Concurrent television and computer usage. Cyberpsychology, Behavior, and Social Networking, 14, 527-534. doi:10.1089/cyber.2010.0350

Cain, M. S., \& Mitroff, S. R. (2011). Distractor filtering in media multitaskers. Perception, 40, 1183-1192. doi:10.1068/p7017

Cain, M. S., \& Mitroff, S. R. (2016). Media multitasking and impulsivity. Manuscript in preparation.

Cain, M. S., Prinzmetal, W., Shimamura, A. P., \& Landau, A. N. (2014). Improved control of exogenous attention in action video game players. Frontiers in Cognition, 5, 69. doi:10.3389/fpsyg.2014.00069

Case, R., Kurland, D. M., \& Goldberg, J. (1982). Operational efficiency and the growth of short-term memory span. Journal of Experimental Child Psychology, 33, 386-404. doi:10.1016/0022-0965(82)90054-6

Cowan, N., Elliott, E. M., Saults, J. S., Morey, C. C., Mattox, S., Hismjatullina, A., \& Conway, A. R. (2005). On the capacity of attention: Its estimation and its role in working memory and cognitive aptitudes. Cognitive Psychology, 51, 42-100. doi:10.1016/j. cogpsych.2004.12.001

De Riddere, G. (2013). Media multitasking and advertising: Friends or foes? The impact of media multitasking and (in)congruency on memory and attitude toward the ad (Masters thesis). University of Ghent, Ghent, Belgium. Retrieved from http://lib.ugent.be/fulltxt/ RUG01/002/064/025/RUG01-002064025_2013_0001_AC.pdf 
Duckworth, A. L., \& Quinn, P. D. (2009). Development and Validation of the Short Grit Scale (Grit-S). Journal of Personality Assessment, 91, 166-174. doi:10.1080/00223890802634290

Duckworth, A. L., Quinn, P. D., \& Tsukayama, E. (2012). What No Child Left Behind leaves behind: The roles of IQ and self-control in predicting standardized achievement test scores and report card grades. Journal of Educational Psychology, 104, 439-451. doi: $10.1037 / \mathrm{a} 0026280$

Duff, B. R. L., Yoon, G., Wang, Z., \& Anghelcev, G. (2014). Doing it all: An exploratory study of predictors of media multitasking. Journal of Interactive Advertising, 14, 11-23. doi:10.1080/15252019.2014.884480

Dweck, C. (2006). Mindset: The new psychology of success. New York, NY: Random House.

Engle, R. W., Tuholski, S. W., Laughlin, J. E., \& Conway, A. R. A. (1999). Working memory, short-term memory, and general fluid intelligence: A latent-variable approach. Journal of Experimental Psychology: General, 128, 309-331. doi:10.1037/0096-3445.128.3.309

Farah, M. J., Shera, D. M., Savage, J. H., Betancourt, L., Giannetta, J. M., Brodsky, N. L., \& Hurt, H. (2006). Childhood poverty: Specific associations with neurocognitive development. Brain Research, 1110, 166-174. doi:10.1016/j.brainres.2006.06.072

Finn, A. S., Kraft, M. A., West, M. R., Leonard, J. A., Bish, C. E., Martin, R. E., \& Gabrieli, J. D. E. (2014). Cognitive skills, student achievement tests, and schools. Psychological Science. doi:10.1177/ 0956797613516008

Foehr, U.G. (2006). Media multitasking among American youth: Prevalence, predictors, and pairings (Report No. 7592). Menlo Park, CA: Kaiser Family Foundation. Retrieved from www.kff. org/entmedia/upload/7592.pdf

Gathercole, S. E., \& Pickering, S. J. (2000). Working memory deficits in children with low achievements in the national curriculum at 7 years of age. British Journal of Educational Psychology, 70, 177-194. doi:10.1348/000709900158047

Gluck, M. A., Shohamy, D., \& Myers, C. (2002). How do people solve the "weather prediction" task? Individual variability in strategies for probabilistic category learning. Learning and Memory, 9, 408-418. doi: $10.1101 / \mathrm{lm} .45202$

Good, C., Aronson, J., \& Inzlicht, M. (2003). Improving adolescents' standardized test performance: An intervention to reduce the effects of stereotype threat. Journal of Applied Developmental Psychology, 24, 645-662. doi:10.1016/j.appdev.2003.09.002

Hackman, D. A., Gallop, R., Evans, G. W., \& Farah, M. J. (2015). Socioeconomic status and executive function: Developmental trajectories and mediation. Developmental Science, 18, 686-702. doi: 10.1111/desc. 12246

Hubert-Wallander, B., Green, C. S., \& Bavelier, D. (2011). Stretching the limits of visual attention: The case of action video games. Wiley Interdisciplinary Reviews: Cognitive Science, 2, 222-230. doi: $10.1002 /$ wcs. 116

John, O. P., \& Srivastava, S. (1999). The Big-Five trait taxonomy: History, measurement, and theoretical perspectives. In L. A. Pervin \& O. P. John (Eds.), Handbook of personality: Theory and research (pp. 102-138). New York, NY: Guilford Press.

Kane, M. J., Conway, A. R. A., Miura, T. K., \& Colflesh, G. J. H. (2007). Working memory, attention control, and the $n$-back task: A question of construct validity. Journal of Experimental Psychology: Learning, Memory, and Cognition, 33, 615-622. doi:10.1037/ 0278-7393.33.3.615

Knowlton, B. J., Squire, L. R., \& Gluck, M. A. (1994). Probabilistic classification learning in amnesia. Learning and Memory, 1, 106-120. doi:10.1101/lm.1.2.106

Kononova, A. (2013). Multitasking across borders: A cross-national study of media multitasking behaviors, its antecedents, and outcomes. International Journal of Communication, 7, 23.
Lawson, G. M., \& Farah, M. J. (2015). Executive function as a mediator between SES and academic achievement throughout childhood. International Journal of Behavioral Development. doi:10.1177/ 0165025415603489

Lin, L., Lee, J., \& Robertson, T. (2011). Reading while watching video: The effect of video content on reading comprehension and media multitasking ability. Journal of Educational Computing Research, 45, 183-201. doi:10.2190/EC.45.2.d

Mackey, A. P., Finn, A. S., Leonard, J. A., Jacoby-Senghor, D. S., West, M. R., Gabrieli, C. F. O., \& Gabrieli, J. D. E. (2015). Neuroanatomical correlates of the income-achievement gap. Psychological Science, 26, 925-933. doi:10.1177/0956797615572233

Minear, M., Brasher, F., McCurdy, M., Lewis, J., \& Younggren, A. (2013). Working memory, fluid intelligence, and impulsiveness in heavy media multitaskers. Psychonomic Bulletin \& Review, 20, 1274-1281. doi:10.3758/s13423-013-0456-6

Ophir, E., Nass, C., \& Wagner, A. D. (2009). Cognitive control in media multitaskers. Proceedings of the National Academy of Sciences, 106, 15583. doi:10.1073/pnas.0903620106

Owen, A. M., McMillan, K. M., Laird, A. R., \& Bullmore, E. (2005). Nback working memory paradigm: A meta-analysis of normative functional neuroimaging studies. Human Brain Mapping, 25, 4659. doi: $10.1002 / \mathrm{hbm} .20131$

Peirce, J. W. (2007). PsychoPy-Psychophysics software in Python. Journal of Neuroscience Methods, 162, 8-13. doi:10.1016/j. jneumeth.2006.11.017

Ralph, B. C. W., Thomson, D. R., Cheyne, J. A., \& Smilek, D. (2014). Media multitasking and failures of attention in everyday life. Psychological Research, 78, 661-669. doi:10.1007/s00426-013-0523-7

Rideout, V. J., Foehr, U. G., \& Roberts, D. F. (2010). Generation $M^{2}$ : Media in the lives of 8-to 18-year-olds (Report No. 8010). Menlo Park, CA: Kaiser Family Foundation. Retrieved from www.kff.org/ entmedia/8010.cfm

Sanbonmatsu, D. M., Strayer, D. L., Medeiros-Ward, N., \& Watson, J. M. (2013). Who multi-tasks and why? Multi-tasking ability, perceived multi-tasking ability, impulsivity, and sensation seeking. PLOS ONE, 8, e54402. doi:10.1371/journal.pone. 0054402

Shohamy, D., Myers, C. E., Hopkins, R. O., Sage, J., \& Gluck, M. A. (2008). Distinct hippocampal and basal ganglia contributions to probabilistic learning and reversal. Journal of Cognitive Neuroscience, 21, 1820-1832. doi: $10.1162 /$ jocn.2009.21138

Soto, C. J., \& John, O. P. (2012). Development of Big Five domains and facets in adulthood: Mean-level age trends and broadly versus narrowly acting mechanisms. Journal of Personality, 80, 881-914. doi:10.1111/j.1467-6494.2011.00752.x

Strobach, T., Frensch, P. A., \& Schubert, T. (2012). Video game practice optimizes executive control skills in dual-task and task switching situations. Acta Psychologica, 140, 13-24. doi:10.1016/j.actpsy. 2012.02.001

Tsukayama, E., Duckworth, A. L., \& Kim, B. (2013). Domain-specific impulsivity in school-age children. Developmental Science, 16, 879-893. doi:10.1111/desc.12067

Wechsler, D. (2003). Wechsler Intelligence Scale for Children (4th ed.). San Antonio, TX: Pearson Education.

West, M. R., Kraft, M. A., Finn, A. S., Martin, R. E., Duckworth, A. L., Gabrieli, C. F. O., \& Gabrieli, J. D. E. (2016). Promise and paradox: Measuring students' non-cognitive skills and the impact of schooling. Educational Evaluation and Policy Analysis, 38, 148-170. doi: $10.3102 / 0162373715597298$

Yap, J. Y., \& Lim, S. W. H. (2013). Media multitasking predicts unitary versus splitting visual focal attention. Journal of Cognitive Psychology, 25, 889-902. doi:10.1080/20445911.2013.835315 